\title{
Forward Collision Probability Evaluation Based on Tired Index of Driver
}

\author{
Yuan-Lin Chen \\ Department of Mechanical Engineering, Ming Chi University of Technology \\ New Taipei City, Taiwan \\ chenyl@mail.mcut.edu.tw
}

\begin{abstract}
This paper presents a forward collision probability evaluation based on tired index of driver for alerting and assisting driver to avoid the forward collision in highway driving. From electroencephalograph (EEG), we could figure out the relaxed index (RI) and focus index (FI) of the driver. And then a tired index (TI) of driver will be found. We use the tired index of driver, the time-to-collision (TTC) and headway time (HT) to be the factors for evaluating the forward collision probability. When we evaluate the forward collision probability indices of TTC and HT, a Mamdani fuzzy inference algorithm is presented for calculating the forward collision probability index of the vehicle. The forward collision probability index (FCPI) is easily understood for the driver even who does not have any professional knowledge in vehicle field. At the same time, the behaviour of driver is taken into account, called the selflearning algorithm, for each of driver using. For the FCPI, the value 0 indicates the $0 \%$ probability of forward collision, and the values 0.5 and 1 indicate the 50\% and $100 \%$ probabilities of forward collision respectively. It is useful and easily understood for the presented probability index of forward collision for alerting driver to avoid the forward collision when driving in highway.
\end{abstract}

Keywords: Electroencephalograph, EEG, Tired Index, Forward Collision Probability Index.

\section{Introduction}

The forward collision is a serious issue in the traffic transportation and there are so many researchers devoted efforts on the forward collision warning technology [1-19]. It is very important to avoid the forward collision in highway driving and this paper presented a concept of forward collision probability based on driver vigilance detection for assisting drivers driving in safety.

Nakaoka et al. [3] focused on a forward collision warning algorithm based on road friction coefficient and driver characteristics. In the paper [4], the objective of this research is to develop an advanced driver assistance system with lane departure warning and forward collision warning functions. The proposed vision-based driver assistance system in this research can detect lane markings and preceding vehicles through a CMOS camera and an image processing unit simultaneously. An approach [8] was proposed to estimate the location, velocity, and acceleration of a target vehicle to avoid a possible collision. Cabrera et al [9] focused on two time-based measures that assess both front and rear collision threats. A common feature of the above methods is that they could not judge how much danger is going on in current driving status. And the driver could not realize the meaning of the factor value which display in the presented collision warning system.

The salient features of this paper are summarized as follows:

- Use TTC factor to evaluate a TTC forward collision probability index.

- Use HT factor for designing a HT forward collision probability index.

- Consider a tired index of driver for designing the TTC and HT forward collision probability index.

- A forward collision probability index called FCPI is presented for warning the driver to avoid the forward collision.

- The self-learning algorithm could figure out the optimal functions of TTC and HT forward collision probability index for each driver used to reduce the wrong warning signal generated.

As mentioned above, the presented method is workable and useful in the forward collision warning system. The experimental results guarantee the performance of the presented method. 


\section{Driving Characteristics Analysis}

In this paper we call 'the driving car' as a car which is driven as follows or host driving and a car in front of the driving car is called 'car ahead'. There are two the most important factors, TTC and HT, which effect the forward collision.

\subsection{Time to Collision, TTC}

The definition of time to collision, TTC, could be expressed as equation (1).

$$
\mathrm{TTC}=\mathrm{D}(\mathrm{t}) / \mathrm{V}_{\mathrm{D}}
$$

where $\mathrm{D}(\mathrm{t})$ is the distance between driving car and the car ahead and the $\mathrm{V}_{\mathrm{D}}$ is a speed between the driving car and the car ahead. $\mathrm{V}_{\mathrm{D}} \leqq 0$ means the driving car is driving in safety condition because the distance between driving car and car ahead becomes larger and larger. In the other hand, when $\mathrm{V}_{\mathrm{D}}>0$ means it will cause a forward collision after TTC time if the driving condition keeps going.

\subsection{Headway Time, HT}

The definition of the headway time, HT, is shown in equation (2). In general case, the value of TTC is greater than or equal to HT. If the speed of vehicle ahead is 0 then the value of TTC will be equal to HT.

$$
\mathrm{HT}=\mathrm{D}(\mathrm{t}) / \mathrm{V}_{\mathrm{F}}
$$

where $\mathrm{V}_{\mathrm{F}}$ is the speed of driving car. If $\mathrm{HT}=1 \mathrm{~s}$ means that the distance between driving car and car ahead is about $27.78 \mathrm{~m}$ under $100 \mathrm{~km} / \mathrm{h}$ speed of the driving car.

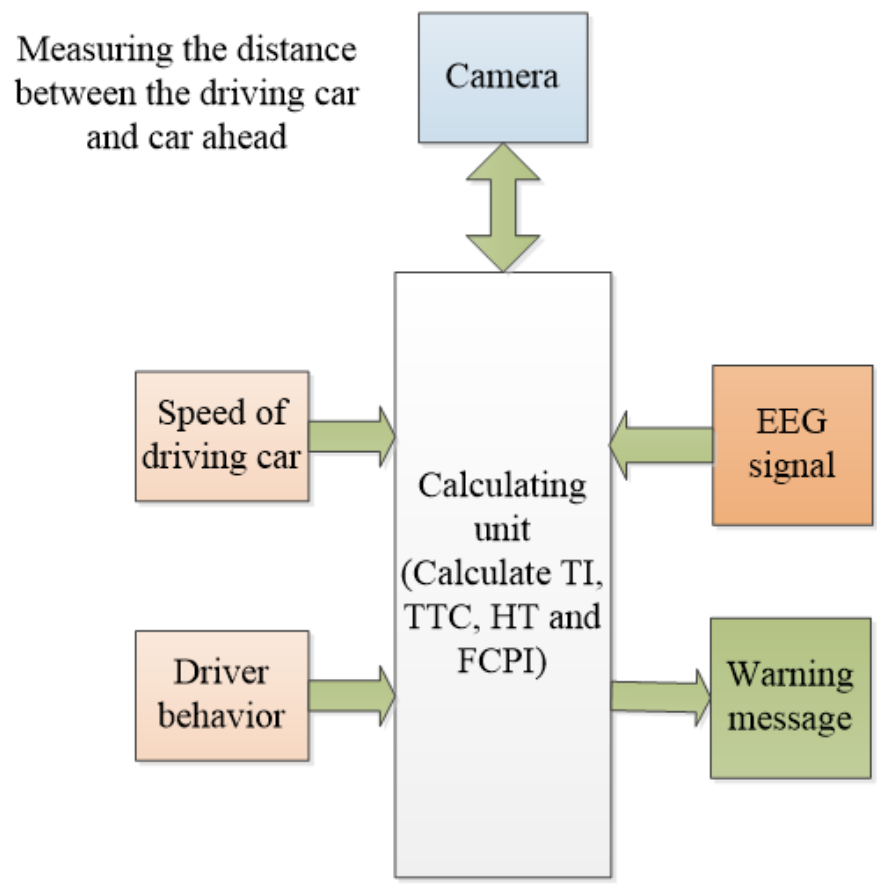

Fig. 1: The block diagram of a forward collision warning system based on the tired index of driver. 


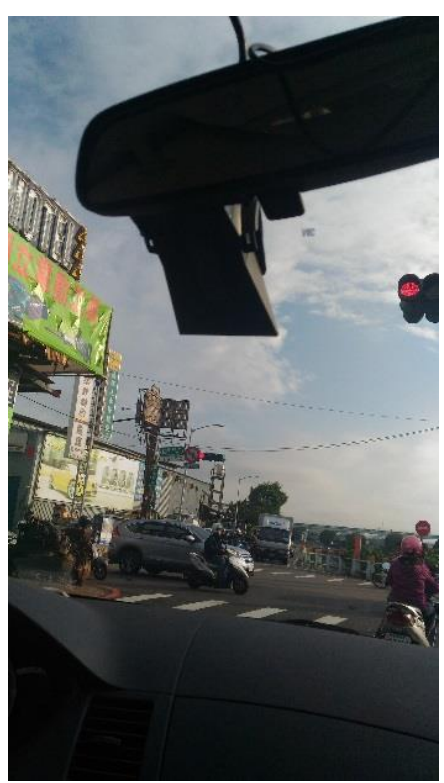

Fig. 2: The camera of C2-270.

\section{A Forward Collision Warning System}

The configuration of presented forward collision warning system based on the tired index of driver is shown in figure 1. We use an equipment model C2-270 [18] to detect the distance between driving car and car ahead. The C2-270 can read the speed of driving car through CAN bus and can use the camera for measuring the distance between the driving car and car ahead. Figures 2 shows the camera which is installed inside the windshield of driving car. The forward collision warning system could read the messages of headway time, speed, brake status, brush status, ..., and so on from the CAN bus of $\mathrm{C} 2-270$.

The presented forward collision warning system could use the headway time, HT, and speed of driving car, $\mathrm{V}_{\mathrm{F}}$, for calculating the value of $\mathrm{D}(\mathrm{t})$ which is the distance between driving car and car ahead. Based on the factors of $\mathrm{HT}, \mathrm{D}(\mathrm{t})$ and $\mathrm{V}_{\mathrm{F}}$, the system could figure out the TTC. After determining the type of membership functions of TTC and HT, then the TTC and HT forward collision probability indices could be calculated based on the tired index of driver. The Mamdanitype fuzzy inference system is used to evaluate the approaching forward collision probability index (AFCPI). The driver could easily understand the meaning of AFCPI which is the risk or how much danger of forward collision probability.

\section{Driver Tired Index}

The tired index (TI) will express the drowsy level of driver. There are many researchers who focus on the drowsiness of drivers [20-28]. In this paper, we use the EEG activities for determining drowsy of driver. An equipment named "brainlink" [29] is used for measuring the TI of driver. The picture of brainlink on the head for EEG measuring is shown in figure 3. The device of brainlink will output the focus index (FI) and relaxed index (RI) of driver by Bluetooth.

The value range of the focus index of driver is between 0 and 100. A zero value measuring means the driver has a very low focus and a higher value of FI means the driver has a high focus in spirit. Another word, we can say that if the driver with a higher FI then the driver is not drowsy. The RI expresses the relaxed level of driver. The value range of RI is same between 0 and 100. A higher value of RI means the driver going into drowsiness situation and a lower value means the driver with in excited condition.

In this paper, we merge the FI and RI to figure out the TI of driver. It's value range between 0 and 100 . A higher value describes the driver with a severe drowsiness. Table 1 shows the description of the FI of driver. In this paper, we use the TI of driver to design the forward collision probability, FCPI. 
Table 1: Description of the TI of driver.

\begin{tabular}{|c|c|l|}
\hline Value of FI & Level & \multicolumn{1}{c|}{ Status of driver } \\
\hline $81 \sim 100$ & Severe drowsiness & $\begin{array}{l}\text { The TI of driver is very high. The driver is falling into a severe drowsiness } \\
\text { situation. We can say the driver in depth drowsy situation. }\end{array}$ \\
\hline $61 \sim 80$ & $\begin{array}{c}\text { Moderate } \\
\text { drowsiness }\end{array}$ & $\begin{array}{l}\text { When the value of TI between 61 and } 80, \text { we determine the driver falling into a } \\
\text { moderate drowsiness status. }\end{array}$ \\
\hline $41 \sim 60$ & Normal & $\begin{array}{l}\text { Normal status of driver, the driver is in normal situation and with a safety } \\
\text { driving action. }\end{array}$ \\
\hline $21 \sim 40$ & Light excited & $\begin{array}{l}\text { When the value of TI between 21 and 40, we could say the driver could be in a } \\
\text { little excited status and response of driving is normal . }\end{array}$ \\
\hline $1 \sim 20$ & Excited & $\begin{array}{l}\text { During this level, we could say the driver in excited status and could not easily } \\
\text { fall into drowsiness shortly. }\end{array}$ \\
\hline
\end{tabular}

\section{Evaluation of Forward Collision Probability}

A Z-shaped membership function $[15,16,19]$ is good designed to model a forward collision probability index which is related to TTC or HT. Figures 3 and 4 show the designing of TTC forward collision probability index (TFCPI) and HT forward collision probability index (HFCPI), respectively. For the Z-shaped membership function in the forward collision problem, the membership value is increased slowly as the TTC/HT decreases toward a value smaller than $\mathrm{TTC}_{\text {set }} / \mathrm{HT}_{\text {set }}$ and the membership value is increased quickly as the TTC/HT decreases toward a critical dangerous value, $\mathrm{TTC}_{\text {critical }} / \mathrm{HT}_{\text {critical }}$, please refer to figures 3 and 4 . This result allows the $\mathrm{TTC} / \mathrm{HT}$ value to be converted to a probability successfully.

The researchers, Zhang et al. [1], presented a three warning levels based on the TTC for forward collision warning system. When TTC smaller than 0.5 second, it is a dangerous situation and a braking commend is needed for avoiding a forward collision. It is a safety driving condition when TTC is greater than 2.5 seconds. It must warn the driver to pay attention when TTC is smaller than 2.5 seconds. In this paper, we set an initial value $\mathrm{TTC}_{\text {critical }}=0.5 \mathrm{~s}$ and an initial value $\mathrm{TTC}_{\text {set }}=2.5 \mathrm{~s}$ as shown in figure 3 . We also present a self-learning algorithm for determining the points $\mathrm{A}$ and $\mathrm{B}$ in figure 3 . The setting points $\mathrm{A}$ and $\mathrm{B}$ are considered the driver behavior for building the forward collision probability index function (please see [16] for more detail).

According to the observation of the paper [12], the point $\mathrm{B}\left(\mathrm{HT}_{\mathrm{set}}\right)$ set to be 1.5 seconds is a good design. When HT is greater than $1.5 \mathrm{~s}$ then the forward collision probability is set to be 0 . It is a safety driving condition when HT is greater than 1.5 seconds. When HT is smaller than 0.3 second, it is the most dangerous situation and the collision probability is set to be $100 \%$ for alerting the driver. It must warn the driver to pay attention when HT is smaller than 1.5 seconds. A membership function to model a HT forward collision probability index is shown in figure 4. Again, we use a self-learning algorithm to determine the points A and B in figure 4 (please see [16] for more detail).

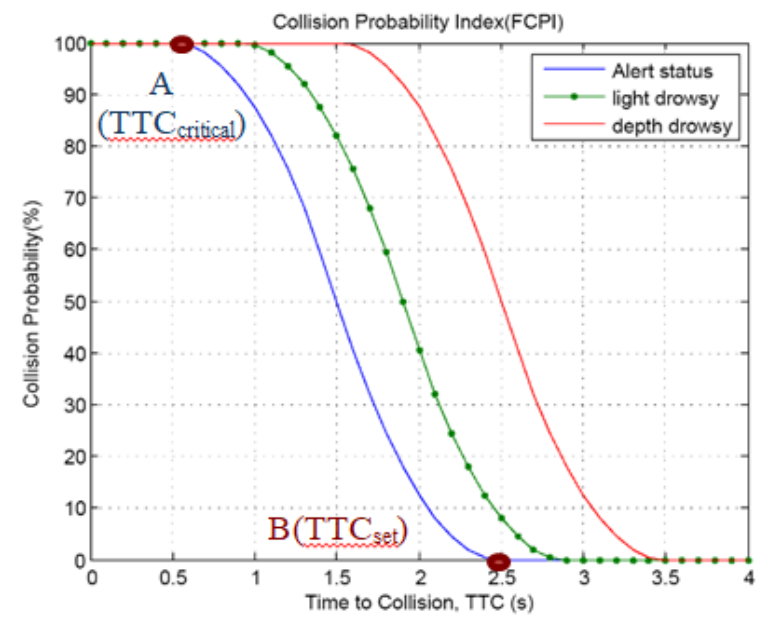

Fig. 3: Function of TTC forward collision probability index based on TI of driver. 


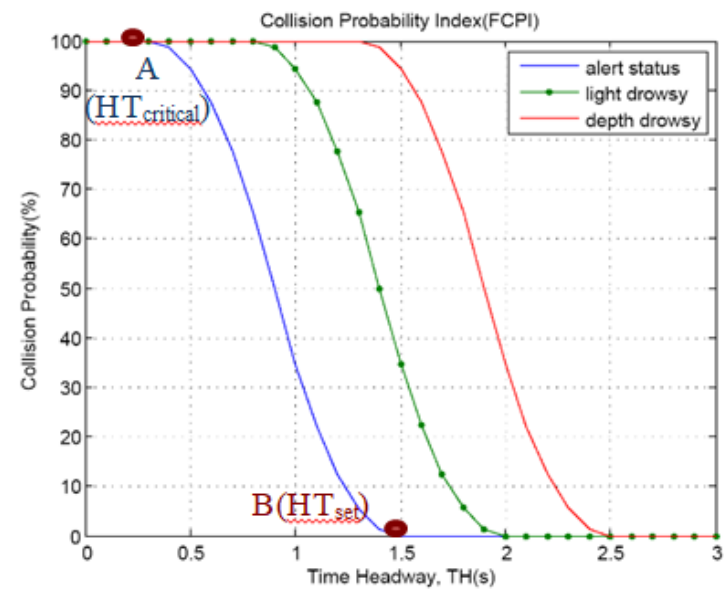

Fig. 4: Function of HT forward collision probability index based on TI of driver.

The Mamdani-type fuzzy inference system [30] is used to evaluate the AFCPI. The equation (4) expresses the AFCPI which combined from TFCPI and HFCPI.

$$
u_{A F C P I}(T T C, H T)=\operatorname{probor}\left[u_{T F C P I}(T T C), u_{H F C P I}(H T)\right]=u_{T F C P I}(T T C)+u_{H F C P I}(H T)-u_{T F C P I}(T T C) u_{H F C P I}(H T)
$$

where $u_{T F C P I}(T T C$ ) is TFCPI (see figure 3$), u_{H F C P I}(H T)$ is HFCPI (see figure 4) and $u_{A F C P I}(T T C, H T$ ) is FCPI.

The TTC and HT are the most important factors in forward collision issue. The equation (3) could merge the effects of TTC and HT successfully. The following examples demonstrate the performance of the AFCPI index.

\section{Experimental Results}

The experimental results guaranteed which is useful for the FCPI based on the TI of driver, considering the driving behavior, are presented in this section.

\subsection{Test Conditions}

- Test car and driver

Test car is a model named Zinger made in Taiwan. The capacity of the engine is 2,400 cc and the net weight of car is $1,690 \mathrm{~kg}$. The driver is a male, 52 years old and has 32 years driving experience.

- Test route

In the paper [16], the test route from Longtan, Taoyuan city to Xinzhuang, New Taipei city in Taiwan was described. The distance of test route between Longtan and Xinzhuang is totally, $35 \mathrm{~km}$. The route includes the Formosa Freeway and Expressway No.65 in Taiwan. Distance on the Formosa Freeway driving, is $25 \mathrm{~km}$ and speed limit is $110 \mathrm{~km} / \mathrm{h}$. The distance on Expressway driving is $10 \mathrm{~km}$ and speed limits are $60 \mathrm{~km} / \mathrm{h}$ and $80 \mathrm{~km} / \mathrm{h}$.

\subsection{Road Driving Test}

- Development display of the presented forward collision warning system

Figure 5 shows a picture of brainlink. For the FCPI forward collision warning system, we design a friendly display for showing the forward collision probability index as shown in figure 6. From right to left, the speed, TFCPI, HFCPI, API probability, TI, FI and RI are shown in figure 6. A moderate warning signal generated on November 4, 2017 driving is shown in figure 6.

\section{- Results of real road testing}

Figure 7 shows the speed curve of the driving vehicle on highway on September 23, 2017, Time started was 17:04:28. The test car was driven on the Expressway No.65 highway before 500 seconds and then drove on Formosa Freeway. The total driving time was 1,560 seconds. 
The HT curve of driving vehicle on September 23, 2017 is shown in figure 8. It is obvious that the driver kept the HT greater than 0.5 second. Over here, if HT is greater than 4 seconds, then HT is equal by $4 \mathrm{~s}$.

Figure 9 shows the results of the TI of driver on road test. A variation of FCPI is shown in figure 10 which indicates the warning forward collision probability. It is obvious that the presented FCPI gives the total 5 times with strong warning. In the analysis, we figure out that the FCPI approaching $100 \%$ was caused by a vehicle cutting into the in front of driving lane.

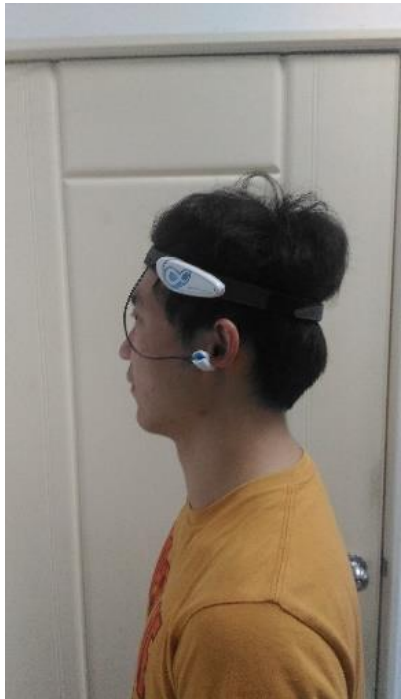

Fig. 5: Picture of brainlink.

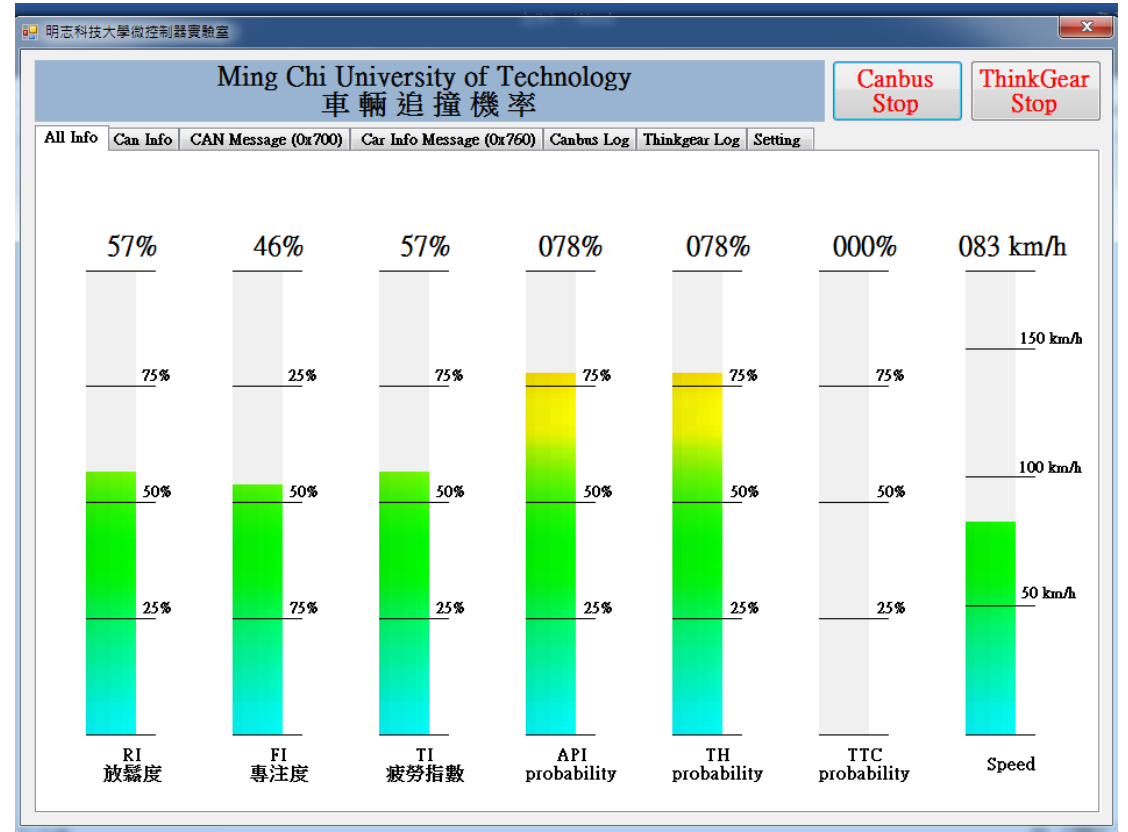

Fig. 6: Display of the presented forward collision warning system (September 23, 2017). 


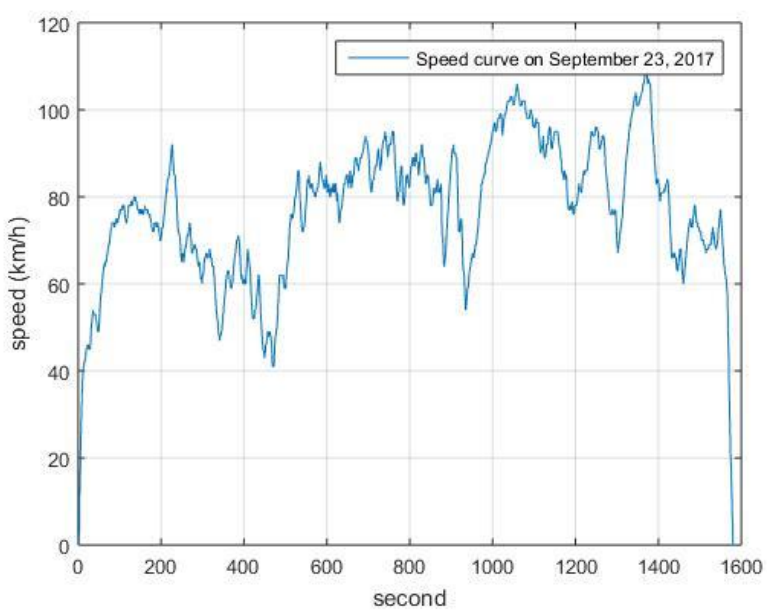

Fig. 7: Speed curve of driving car on September 23, 2017.

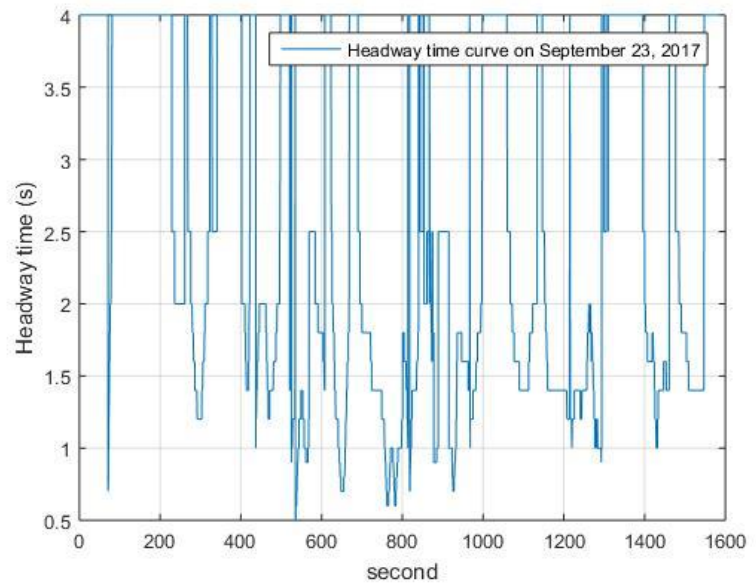

Fig. 8: HT curve of driving car on September 23, 2017.

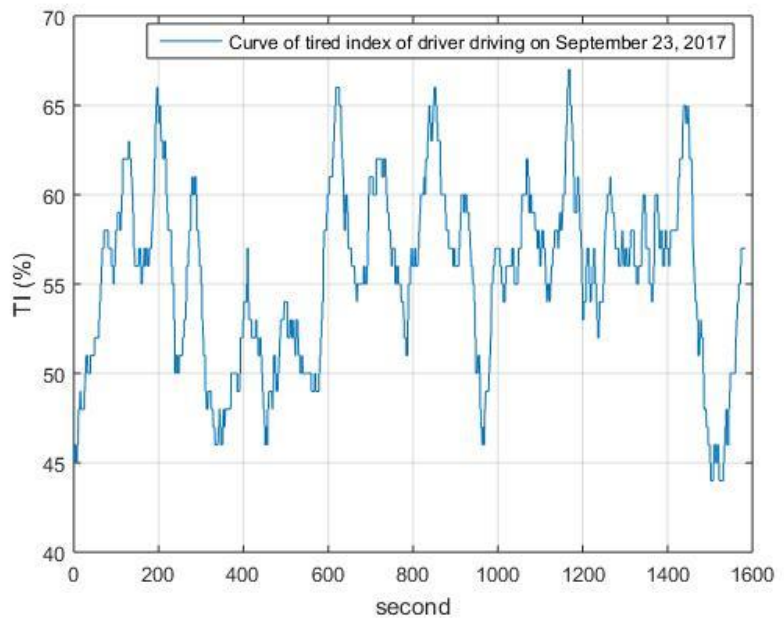

Fig. 9: TI curve of driver on September 23, 2017. 


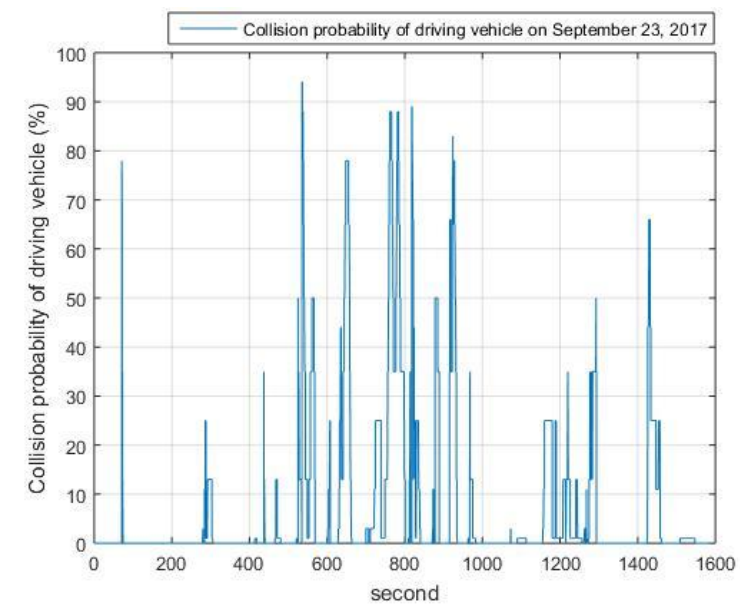

Fig. 10: Variation of FCPI of driving car on September 23, 2017.

The performance of using the presented FCPI with considering the tired index of driver in forward collision warning system is guaranteed. The experimental results described the presented method is suitable applying in forward collision warning system for avoiding the forward collision accident.

\section{Conclusion}

A forward collision probability index considering the tired index of driver for forward collision warning system is presented in this paper for alerting the driver to avoid a forward collision on highway driving. A 100\% value of a forward collision probability index indicates the forward collision will happen and in some special case it will not occur but close to occur. A lager value of FCPI means the driving car has a higher probability to cause a forward collision. The presented index which calculation is based on the self-learning algorithm could be realized clearly for drivers even the driver who doesn't have any professional knowledge regarding vehicle engineering field. The tired index of driver is taken into account. The experimental results described the presented index FCPI is suitable applying in forward collision warning system and warning driver avoiding a forward collision is guaranteed. The FCPI based on tired index of driver can be served as a safety driving assistance system for safety driving on highway.

\section{Acknowledgements}

This work was supported by the Ministry of Science and Technology of Taiwan for financial support under contract number MOST 105-2221-E-131-005.

\section{References}

[1] Y. Zhang, E. K. Antonsson and K. Grote, "A New Threat Assessment Measure for Collision Avoidance Systems," 2006 IEEE Intelligent Transportation Systems Conference, Toronto, Canada, pp.968-975, 2006.

[2] A. Lindgren, F. Chen, P. W. Jordan, and H. Zhang, "Requirements for the design of advanced driver assistance systems," Int. J. Design, vol. 2, no. 2, pp. 41-54, 2008.

[3] M. Nakaoka, P. Raksincharoensak and M. Nagai, "Study on Forward Collision Warning System Adapted to Driver Characteristics and Road Environment," International Conference on Control, Automation and Systems 2008 in COEX, Seoul, Korea, pp. 2890-2895, 2008.

[4] J.-F. Liu, Y.-F. Su, M.-K. Ko, P.-N. Yu, "Development of a Vision-Based Driver Assistance System with Lane Departure Warning and Forward Collision Warning Functions," Computing: Techniques and Applications, DICTA'08. Digital Image, pp. 480-485, 2008.

[5] E. Dagan, O. Mano, G. P. Stein, A. Shashua, "Forward Collision Warning with a Single Camera," 2004 IEEE Intelligent Vehicles Symposium, Parma, Italy, pp. 37-42, 2004.

[6] S.-K. Chen, and J. S. Parikh, "Developing a Forward Collision Warning System Simulation," IEEE Intelligent Vehicles Symposium 2000, Dearborn (MI), USA, pp. 338-343, 2000. 
[7] D. F. Llorca, M. A. Sotelo, I. Parra, J. E. Naranjo, M. Gavilán, and S. Álvarez, "An Experimental Study on Pitch Compensation in Pedestrian-Protection Systems for Collision Avoidance and Mitigation," IEEE Transactions on Intelligent Transportation Systems, vol. 10, no. 3, pp. 469-474, 2009.

[8] P.-J. Tu and J.-F. Kiang, "Estimation on Location, Velocity, and Acceleration with High Precision for Collision Avoidance," IEEE Transactions on Intelligent Transportation Systems, vol. 11, no. 2, pp.374-379, 2010.

[9] A. Cabrera, S. Gowal and A. Martinoli, "A New Collision Warning System for Lead Vehicles in Rear-end Collisions," 2012 Intelligent Vehicles Symposium, Alcalá de Henares, Spain, pp.674-679, 2012.

[10] Y.-L. Chen, "An Approaching Index to Evaluate a forward Collision Probability," ICITSA 2017: 19th International Conference on Intelligent Transport Systems and Applications, Chicago, USA, 2017.

[11] N. Ovcharova, M. Fausten, F. Gauterin, "Effectiveness of Forward Collision Warnings for Different Driver," 2012 Intelligent Vehicles Symposium, Alcalá de Henares, Spain, pp. 944-949, 2012.

[12] J. Wang, L. Zhang, D. Zhang, and K. Li, "An Adaptive Longitudinal Driving Assistance System Based on Driver Characteristics," IEEE Transactions on Intelligent Transportation Systems, vol. 14, no. 1, pp.1-12, 2013.

[13] Y.-L. Chen, K.-Y. Shen, S.-C. Wang, "Forward Collision Warning System Considering Both Time-to-Collision and Safety Braking Distance," Int. J. Vehicle Safety, vol. 6, no. 4, pp. 347-360, 2013.

[14] Y.-L. Chen, and W.-J. Lee, "Safety Distance Warning System with a Novel Algorithm for Vehicle Safety Braking Distance Calculating," International Journal of Vehicle Safety, vol. 5, no. 3, pp. 213-231, 2011.

[15] Yuan-Lin Chen, "Study on A Novel Forward Collision Probability Index", Int. J. Vehicle Safety, Vol. 8, No. 3, 2015, pp.193-204.

[16] Y.-L. Chen, "Using the Driving Behavior to Design a Forward Collision Probability Index," Int. J. Vehicle Safety, vol. 9, no. 3, pp.196-208, 2017.

[17] L. Li, G. Lu, Y. Wang and D. Tian, “A Rear-end Collision Avoidance System of Connected Vehicles," 2014 IEEE 17th International Conference on Intelligent Transportation Systems (ITSC), Qingdao, China, pp. 63-68, 2014.

[18] Mobileye company. [Online]. Available: http://www.mobileye.com

[19] Y.-L. Chen, "Using an Approaching Index to Evaluate a Forward Collision Probability," submitted to IET Intelligent Transport Systems, 2017.

[20] C. T. Lin, C. J. Chang, B. S. Lin, S. H. Hung, C. F. Chao, I. J. Wang, "A real-time wireless brain-computer interface system for drowsiness detection," IEEE Trans. Biome. Circuits Syst., vol. 4, no. 4, pp. 214-222, 2010.

[21] A. Picot, S. Charbonnier, and A. Caplier, "On-line detection of drowsiness using brain and visual information," IEEE Trans. Syst., Man, Cybern. A, Syst., Humans, vol. 42, no. 2, pp. 764-775, May. 2012.

[22] J. W. Lee, S. K. Lee, C. H. Kim, K. H. Kim, O. C. Kwon, "Detection of drowsy driving based on driving information," in Proc. Int. ICTC, pp. 607-608, 2014.

[23] R. N. Khushaba, S. Kodagoda, S. Lal, and G. Dissanayake, "Driver drowsiness classification using fuzzy waveletpacket-based feature-extraction algorithm," IEEE Trans. Biomed. Eng., vol. 58, no. 1, pp. 121-131, 2011.

[24] L. M. Bergasa, J. Nuevo, M. A. Sotelo, M. Vazquez, "Real-time system for monitoring driver vigilance," IEEE Trans. Intell. Transp. Syst., vol. 7, no. 1, pp. 63-77, 2006.

[25] C. T. Lin, Y. C. Chen, T. Y. Huang, T. T. Chiu, L. W. Ko, S. F. Liang, H. Y. Hsieh, S. H. Hsu, J. R. Duann, "Development of wireless brain computer interface with embedded multitask scheduling and its application on realtime driver's drowsiness detection and warning," IEEE Trans. Biomed. Eng., vol. 55, no. 5, pp. 1582-1591, 2008.

[26] C. T. Lin, C. H. Chuang, C. S. Huang, S. F. Tsai, S. W. Lu, Y. H. Chen, L. W. Ko, "Wireless and wearable EEG system for evaluating driver vigilance," IEEE Trans. Biomed. Circuits Syst., vol. 8, no. 2, pp. 165-176, 2014.

[27] F. C. Lin, L. W. Ko, C. H. Chuang, T. P. Su, C. T. Lin, "Generalized EEG-based drowsiness prediction system by using a self-organizing neural fuzzy system," IEEE Trans. Circuits Syst. I, Reg Papers, vol. 59, no. 9, pp. 20442055, 2012.

[28] H. Yu, H. Lu, T. Ouyang, H. Liu, B. L. Lu, "Vigilance detection based on sparse representation of EEG," in Proc. IEEE Ann. Int. EMBC, pp. 2439-2442, 2010.

[29] Sheng Hong INC., [Online]. Available: www.brain-sh.tw/

[30] G. Sánchez-Torrubia, C. Torres-Blanc, "A Mamdani-Type Fuzzy Inference System To Automatically Assess Dijkstra's Algorithm Simulation," International Journal "Information Theories and Applications, vol. 17, no. 1, pp.35-100, 2010. 